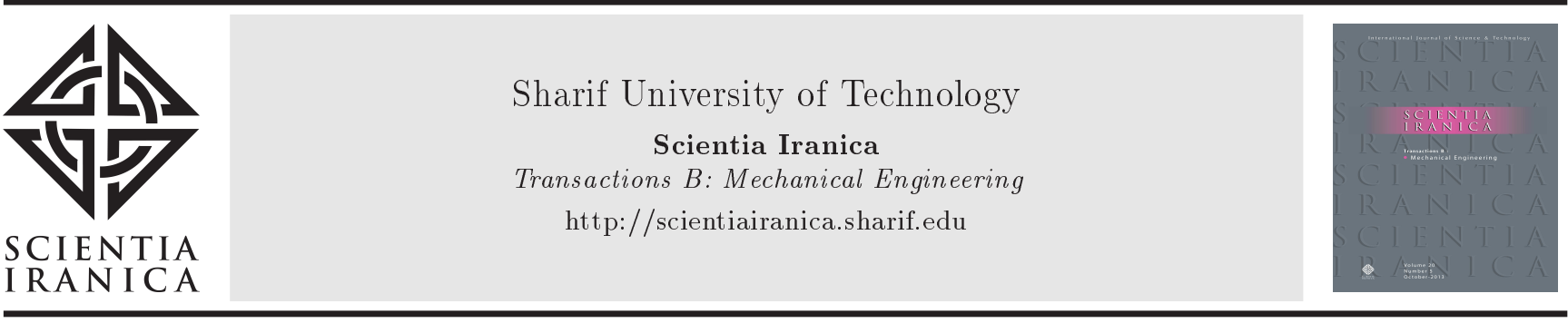

\title{
Adaptive dynamic surface control of a flexible-joint robot with parametric uncertainties
}

\author{
C.G. Li*, W. Cui, D.D. Yan, Y. Wang, and C.M. Wang \\ College of Mechanical and Electrical Engineering, Nanjing University of Aeronautics and Astronautics, Nanjing, 210016, China.
}

Received 8 January 2017; received in revised form 12 February 2018; accepted 14 May 2018

\author{
KEYWORDS \\ FJ robot; \\ Dynamic surface \\ control; \\ Inertial parameters; \\ Adaptive control; \\ Tracking accuracy.
}

\begin{abstract}
A new kind of adaptive Dynamic Surface Control (DSC) method is proposed in this study to overcome parametric uncertainties of Flexible-Joint (FJ) robots. These uncertainties of FJ robots are transformed into linear expressions of inertial parameters, which are estimated based on the DSC, and the high-order derivatives in DSC are solved by using a first-order filter. The adaptation laws of inertial parameters are designed directly to improve the tracking performance according to the Lyapunov stability analysis. Simulation results of a two-link FJ robot show better tracking accuracy against model parametric uncertainties. The used method does not need the aid of Neural Network (NN); it is simpler and calculates faster than the other adaptive methods.
\end{abstract}

(C) 2019 Sharif University of Technology. All rights reserved.

\section{Introduction}

Flexible-Joint (FJ) robots equipped with harmonic drivers, windup shaft, and force/torque sensors are used widely in the areas such as aerospace, service robots, and so on [1-3]. Because of the uncertain dynamic parameters such as inertia, flexibility, and the external disturbances, the tracking performance of robots is not ideal generally and, even, the systems are unstable under basic control methods.

In the last decades, many researchers have proposed and developed the backstepping control technique because of its advantages such as systematic and recursive design methodology for nonlinear control. However, its disadvantage is "explosion of complexity" or "explosion of derivative" caused by the repeated differentiation of virtual control vectors, resulting in

\footnotetext{
*. Corresponding author. Tel.: 862584892503

E-mail addresses: lichenggang@nuaa.edu.cn (C.G. Li); 876399207@qq.com (W.Cui);

1024849527@qq.com (D.D.Yan); 948390245@qq.com (Y. Wang); $1048518650 @ q q . c o m$ (C.M.Wang)
}

doi: $10.24200 /$ sci. 2018.20492 heavy computing burden $[4,5]$. To overcome this complexity, the DSC is investigated by introducing a firstorder filter in the backstepping procedure [6]. Because of the great approximation capability of nonlinear functions, the Neural Networks (NNs) are widely utilized in the control systems to compensate the uncertainties of parameter and are combined with the DSC and backstepping techniques to design the controller [714]. In [14], NN adaptive backstepping controller was proposed, and Radial Basic Function (RBF) was used to approximate the nonlinear unknowns in the backstepping design. Simulation results showed good tracking performance.

Due to the adaptiveness of fuzzy, it was employed too in the control design [15-19]. In [15], the backstepping control design was suggested, and the adaptive fuzzy DSC was applied to approximate unknown nonlinear control system, which was more general for practical applications in the presence of input saturation. The prescribed performance of switched adaptive DSC was investigated for a class of switched nonlinear systems, and mode-dependent fuzzy logic systems were used to approximate the switching nonlinear functions [16]. The adaptive fuzzy 
dynamic surface control was investigated for a class of nonlinear systems with fuzzy dead zone and unmodeled dynamics. The Takagi-Sugeno-type fuzzy logic systems were adopted to approximate the unknown functions in system [17]. [20] employed the Fuzzy Neural Networks (FNNs) to approximate uncertain dynamics and bounding functions when the active DSC was developed to suppress regenerative chatter in micromilling.

Some disturbance oservers (DOB) are employed commonly to design nonlinear controllers because of their distinct physical meaning [21-23]. In [21], a GainScheduled Dynamic Surface Control (GSDSC) based on the NN disturbance observer (NNDOB) was proposed for a class of uncertain underactuated mechanical systems, which efficiently solves the mismatches and overcomes the problem of "explosion of complexity". In [22], a nonlinear disturbance observer was constructed to overcome the unknown environmental disturbances; then, a controller was designed based on the combination of backstepping and DSC techniques. An observer-based DSC scheme was developed to overcome the problems of model uncertainties, unsteady aerodynamics, and actuator saturation in [23].

Sliding Mode Control (SMC) is one of the efficient control schemes for compensating external disturbances and parametric uncertainties. In [24,25], sliding mode control and adaptive control were introduced into the backstepping control technique together with DSC.

Further, [26] designed an adaptive backstepping controller with a dynamic surface method to solve the problem of parameters of permanent-magnet synchronous motor; the particle swarm optimization algorithm was adopted to adjust and determine the control parameters.

Although the above approximate control schemes enjoy a number of advantages, they are susceptible to certain problems such as heavy calculation burden, long training time, approximating error, and so on, resulting in the difficulty choosing the control parameters and guaranteeing system stability.

In [27], the dynamic surface adaptive backstepping control scheme was employed to prevent the uncertainties of nonlinear dynamics of aircraft. By changing the inertial parameters and positions of aircraft center of gravity, the simulation showed the effectiveness of the controller. However, the selected parameters were limited and inflexible.

In this paper, a new adaptive DSC technique for FJ robots is proposed to overcome the problems of parametric uncertainties, explosion of derivative, and calculation inefficiency. First, model uncertainties of FJ robots are expressed as linear equations of its inertial parameters; thus, the model uncertainties are transformed to compute the evaluation of inertial parameters. The controller is designed by backstepping and adaptive DSC techniques. Then, the adaptation laws for these inertial parameters are derived according to the Lyapunov stability analysis, which reduce the computing burden efficiently. Finally, simulations of the two-link FJ robot show the effectiveness of the adaptive DSC technique.

This paper is organized as follows. In Section 2, the basic dynamic models and properties of FJ robot are introduced. In Section 3, the backstepping control design method is proposed according to the Lyapunov stability theorem. Simulation results and analysis are discussed in Section 4. Finally, some conclusions are given in Section 5 .

\section{Dynamic model of $\mathbf{F J}$ robots}

In general, the dynamic model of $n$-link FJ robot consists of robot dynamics and actuator dynamics, which can be expressed in the following form [28]:

$$
\begin{aligned}
& \mathbf{D}(\mathbf{q}) \ddot{\mathbf{q}}+\mathbf{C}(\mathbf{q}, \dot{\mathbf{q}}) \dot{\mathbf{q}}+\mathbf{G}(\mathbf{q})+\mathbf{K}\left(\mathbf{q}-\mathbf{q}_{m}\right)=\mathbf{0}, \\
& \mathbf{J} \ddot{\mathbf{q}}_{m}+\mathbf{K}\left(\mathbf{q}_{m}-\mathbf{q}\right)=\mathbf{u},
\end{aligned}
$$

where $\mathbf{q}, \dot{\mathbf{q}}, \ddot{\mathbf{q}} \in R^{n}$ denote the position, velocity, and acceleration vectors of the links, respectively, $\mathbf{D}(\mathbf{q}) \in$ $R^{n}$ denotes the inertia matrix, $\mathbf{C}(\mathbf{q}, \dot{\mathbf{q}}) \in R^{n}$ is the Coriolis and centripetal matrix, $\mathbf{G}(\mathbf{q}) \in R^{n}$ denotes the gravity matrix, $\mathbf{K} \in R^{n}$ represents a positive definite diagonal constant flexibility matrix, $\mathbf{q}_{m}, \ddot{\mathbf{q}}_{m} \in R^{n}$ denote the position and acceleration vectors for the actuator, respectively, $\mathbf{J} \in R^{n}$ is the actuator inertia matrix, and $\mathbf{u} \in R^{n}$ is the actual control vector of the actuator torque. In dynamic equations (Eqs. (1) and (2)), the joint stiffness terms are assumed to be dominant among all of the system parameters, and the joint damping ones are neglected. As introduced in literature [9], these equations have a few fundamental properties to make the control system design simple. These properties are presented below.

- (P1): The link inertia matrix, $\mathbf{D}(\mathbf{q})$, is positive definite, symmetric and bounded:

$$
D_{m} \leq\|\mathbf{D}(\mathbf{q})\| \leq D_{M}
$$

where $D_{m}$ and $D_{M}$ denote two different positive constants, respectively.

- (P2): The Coriolis and centripetal matrix, $\mathbf{C}(\mathbf{q}, \dot{\mathbf{q}})$, and gravity matrix, $\mathbf{G}(\mathbf{q})$, are bounded, respectively:

$$
\|\mathbf{C}(\mathbf{q}, \dot{\mathbf{q}})\| \leq C_{M}, \quad\|\mathbf{G}(\mathbf{q})\| \leq G_{M},
$$

where $D_{m}$ and $D_{M}$ denote positive constants.

- (P3): The matrix $\dot{\mathbf{D}}(\mathbf{q})-2 \mathbf{C}(\mathbf{q}, \dot{\mathbf{q}})$ is skewsymmetric. For any vector $\mathbf{x}$,

$$
\mathbf{x}^{T}(\dot{\mathbf{D}}(\mathbf{q})-2 \mathbf{C}(\mathbf{q}, \dot{\mathbf{q}})) \mathbf{x}=\mathbf{0} .
$$


- (P4): The system parameters can be linear in the equation expressed as coefficients of known functions of $\mathbf{q}, \dot{\mathbf{q}}$ and $\ddot{\mathbf{q}}$ for a rigid joint robot. Therefore, the left-hand side of Eq. (1) can be expressed as follows:

$$
\mathbf{D}(\mathbf{q}) \ddot{\mathbf{q}}+\mathbf{C}(\mathbf{q}, \dot{\mathbf{q}}) \dot{\mathbf{q}}+\mathbf{G}(\mathbf{q})=\mathbf{Y}(\mathbf{q}, \dot{\mathbf{q}}, \ddot{\mathbf{q}}) \theta_{1}
$$

where $\mathbf{Y}(\mathbf{q}, \dot{\mathbf{q}}, \ddot{\mathbf{q}}) \in \mathbf{R}^{n \times r}$ denotes the regression matrix, $\theta_{1} \in \mathbf{R}^{r \times 1}$ is an $r$-dimensional vector of parameters, and $r$ is the number of inertia parameters.

Further, Eq. (4) can be rewritten according to the literature [29] as follows:

$$
\mathbf{D}(\mathbf{q}) \mathbf{a}_{1}+\mathbf{C}(\mathbf{q}, \dot{\mathbf{q}}) \mathbf{v}_{1}+\mathbf{G}(\mathbf{q})=\mathbf{Y}\left(\mathbf{q}, \dot{\mathbf{q}}, \mathbf{v}_{1}, \mathbf{a}_{1}\right) \theta_{1}
$$

where $\mathbf{a}_{1}$ and $\mathbf{v}_{1}$ are the arbitrary $n$-dimensional vectors.

As a preliminary to the control design, $\mathbf{x}_{1}=\mathbf{q}_{m}$ and $\mathbf{x}_{2}=\dot{\mathbf{q}}_{m}$ are defined as the functions of state space variables, and dynamic systems (1) and (2) are described as follows:

$$
\begin{aligned}
& \mathbf{D}(\mathbf{q}) \ddot{\mathbf{q}}+\mathbf{C}(\mathbf{q}, \dot{\mathbf{q}}) \dot{\mathbf{q}}+\mathbf{G}(\mathbf{q})+\mathbf{K q}=\mathbf{K} \mathbf{x}_{1}, \\
& \dot{\mathbf{x}}_{1}=\mathbf{x}_{2}, \\
& \dot{\mathbf{x}}_{2}=\mathbf{J}^{-1} \mathbf{u}-\mathbf{J}^{-1} \mathbf{K}\left(\mathbf{x}_{1}-\mathbf{q}\right) .
\end{aligned}
$$

To develop the controller for systems (6)-(8), some assumptions $[8,22]$ are proposed:

Assumption 1. All the state space variables can be achieved by some sensors.

Assumption 2. The model parameters of $\mathbf{D}(\mathbf{q})$, $\mathbf{C}(\mathbf{q}, \dot{\mathbf{q}}), \mathbf{G}(\mathbf{q}), \mathbf{K}$ in Eq. (6) are unknown. However, there are positive constants $K_{m}, K_{M}, J_{m}, J_{M}$ to make

$$
K_{m} \leq\|\mathbf{K}\| \leq K_{M}, \quad J_{m} \leq\|\mathbf{J}\| \leq J_{M} .
$$

Assumption 3. The desired trajectories of link position $\mathbf{q}_{d}$ are uniformly bounded, differentiable up to the second order and satisfy the inequality:

$$
\left\|\mathbf{q}_{d}\right\|^{2}+\left\|\dot{\mathbf{q}}_{d}\right\|^{2}+\left\|\ddot{\mathbf{q}}_{d}\right\|^{2} \leq \mathbf{Q}
$$

where $\mathbf{Q}$ is the positive constant.

\section{Adaptive DSC system}

\subsection{Controller design}

The system inertia parameters in FJ robots are classified into four vectors: $\boldsymbol{\theta}_{1}, \boldsymbol{\theta}_{2}, \boldsymbol{\theta}_{3}$, and $\boldsymbol{\theta}_{4}$, which are composed of the elements in the link dynamic parametric matrix, the inverse of joint stiffness matrix, the joint stiffness matrix, and the actuator inertia matrix, respectively.
Thus, the parametric uncertainties regarding the robot model that include $\mathbf{D}(\mathbf{q}), \mathbf{C}(\mathbf{q}, \dot{\mathbf{q}}), \mathbf{G}(\mathbf{q}), \mathbf{K}^{-1}$, $\mathbf{K}$, and $\mathbf{J}$ can be expressed and solved by using the vectors $\boldsymbol{\theta}_{i}(i=1,2,3,4)$.

Here, the adaptive DSC algorithm is proposed according to the backstepping control design [4], and the design procedure includes three phases:

1. Designing the virtual control vector, $\overline{\boldsymbol{\alpha}}_{1}$, for actuator position $\mathbf{x}_{1}$;

2. Designing the virtual control vector, $\overline{\boldsymbol{\alpha}}_{2}$, for actuator velocity $\mathbf{x}_{2}$;

3. Designing the actual control vector, u. Details are shown as follows:

\section{Phase 1}

Choose a virtual control vector $\overline{\boldsymbol{\alpha}}_{1}$ for $\mathbf{x}_{1}$ as follows:

$$
\overline{\boldsymbol{\alpha}}_{1}=\mathbf{q}+\hat{\mathbf{K}}^{-1} \mathbf{u}_{r},
$$

where $(\hat{\bullet})$ is the estimated value of $(\bullet)$, and the auxiliary control vector, $\mathbf{u}_{r}$, is defined as follows:

$$
\mathbf{u}_{r}=\hat{\mathbf{D}}(\mathbf{q}) \mathbf{a}_{1}+\hat{\mathbf{C}}(\mathbf{q}, \dot{\mathbf{q}}) \mathbf{v}_{1}+\hat{\mathbf{G}}(\mathbf{q})-\boldsymbol{\Lambda}_{2} \mathbf{r}_{1},
$$

where $\mathbf{v}_{1}=\dot{\mathbf{q}}_{d}-\mathbf{\Lambda}_{1} \tilde{\mathbf{q}}, \mathbf{a}_{1}=\dot{\mathbf{v}}_{1}, \mathbf{r}_{1}=\dot{\mathbf{q}}-\mathbf{v}_{1}=$ $\dot{\tilde{\mathbf{q}}}+\boldsymbol{\Lambda}_{1} \tilde{\mathbf{q}}, \tilde{\mathbf{q}}=\mathbf{q}-\mathbf{q}_{d}, \boldsymbol{\Lambda}_{1} \in \mathbf{R}^{n \times n}$ and $\boldsymbol{\Lambda}_{2} \in \mathbf{R}^{n \times n}$ are diagonal positive definite matrices [16], $\mathbf{q}_{d}$ is the desired trajectory of link position.

Eq. (10) can be rewritten by using (P4) as follows:

$$
\mathbf{u}_{r}=\mathbf{Y}\left(\mathbf{q}, \dot{\mathbf{q}}, \mathbf{v}_{1}, \mathbf{a}_{1}\right) \hat{\boldsymbol{\theta}}_{1}-\mathbf{\Lambda}_{2} \mathbf{r}_{1} \text {. }
$$

To avoid the problem of "explosion of derivative", the estimated value of derivative of $\overline{\boldsymbol{\alpha}}_{1}$ is derived by using the first-order filter in the DSC. That is:

$$
\tau_{1} \dot{\boldsymbol{\alpha}}_{1}+\boldsymbol{\alpha}_{1}=\overline{\boldsymbol{\alpha}}_{1}, \quad \boldsymbol{\alpha}_{1}(0)=\overline{\boldsymbol{\alpha}}_{1}(0),
$$

where $\boldsymbol{\tau}_{1}$ denotes a diagonal positive define matrix, and $\boldsymbol{\alpha}_{1}$ is the filtering control vector.

Define:

$$
\mathbf{z}_{1}=\mathbf{x}_{1}-\alpha_{1}
$$

By substituting Eq. (13) into Eq. (6), it can be obtained as follows:

$$
\mathbf{D}(\mathbf{q}) \ddot{\mathbf{q}}+\mathbf{C}(\mathbf{q}, \dot{\mathbf{q}}) \dot{\mathbf{q}}+\mathbf{G}(\mathbf{q})+\mathbf{K q}=\mathbf{K} \alpha_{1}+\mathbf{K} \mathbf{z}_{1} .
$$

From Eqs. (9), (12), and (14), the following is yielded:

$$
\begin{aligned}
\mathbf{D}(\mathbf{q}) \ddot{\mathbf{q}} & +\mathbf{C}(\mathbf{q}, \dot{\mathbf{q}}) \dot{\mathbf{q}}+\mathbf{G}(\mathbf{q})+\mathbf{K q} \\
& =\mathbf{K} \mathbf{q}+\mathbf{K} \hat{\mathbf{K}}^{-1} \mathbf{u}_{r}+\mathbf{K} \mathbf{z}_{1}-\mathbf{K} \boldsymbol{\tau}_{1} \dot{\boldsymbol{\alpha}}_{1} \\
& =\mathbf{K q}+\mathbf{u}_{r}+\mathbf{K}\left(\hat{\mathbf{K}}^{-1}-\mathbf{K}^{-1}\right) \mathbf{u}_{r} \\
& +\mathbf{K} \mathbf{z}_{1}-\mathbf{K} \boldsymbol{\tau}_{1} \dot{\boldsymbol{\alpha}}_{1} .
\end{aligned}
$$

To get the estimated value of $\hat{\mathbf{K}}^{-\mathbf{1}}$, the definition is given by: 


$$
\hat{\mathbf{K}}^{-1} \mathbf{u}_{r}=\operatorname{diag}\left(\mathbf{u}_{r}\right) \hat{\boldsymbol{\theta}}_{2},
$$

where $\operatorname{diag}(\bullet)$ denotes the diagonal matrix composed of all the elements of $(\bullet)$. yields:

Then, substituting Eq. (16) and (P4) into Eq. (15)

$$
\begin{aligned}
\mathbf{D}(\mathbf{q}) \dot{\mathbf{r}}_{1} & +\mathbf{C}(\mathbf{q}, \dot{\mathbf{q}}) \mathbf{r}_{1}+\boldsymbol{\Lambda}_{2} \mathbf{r}_{1}=\tilde{\mathbf{D}}(\mathbf{q}) \mathbf{a}_{1}+\tilde{\mathbf{C}}(\mathbf{q}, \dot{\mathbf{q}}) \mathbf{v}_{1} \\
& +\tilde{\mathbf{G}}(\mathbf{q})+\mathbf{K} \operatorname{diag}\left(\mathbf{u}_{r}\right) \tilde{\boldsymbol{\theta}}_{2}+\mathbf{K} \mathbf{z}_{1}-\mathbf{K} \boldsymbol{\tau}_{1} \dot{\boldsymbol{\alpha}}_{1} \\
& =\mathbf{Y}\left(\mathbf{q}, \dot{\mathbf{q}}, \mathbf{v}_{1}, \mathbf{a}_{1}\right) \tilde{\boldsymbol{\theta}}_{1}+\mathbf{K} \operatorname{diag}\left(\mathbf{u}_{r}\right) \tilde{\boldsymbol{\theta}}_{2}+\mathbf{K} \mathbf{z}_{1} \\
& -\mathbf{K} \boldsymbol{\tau}_{1} \dot{\boldsymbol{\alpha}}_{1}
\end{aligned}
$$

where $(\tilde{\bullet})=(\bullet)-(\hat{\bullet})$.

From Eqs. (9) and (16), the equation can be described as follows:

$$
\overline{\boldsymbol{\alpha}}_{1}=\mathbf{q}+\operatorname{diag}\left(\mathbf{u}_{r}\right) \hat{\boldsymbol{\theta}}_{2}
$$

\section{Phase 2}

Differentiating Eq. (13) yields:

$$
\dot{\mathrm{z}}_{1}=\dot{\mathrm{x}}_{1}-\dot{\alpha}_{1}=\mathrm{x}_{2}-\dot{\alpha}_{1} .
$$

Design the second virtual control vector $\overline{\boldsymbol{\alpha}}_{2}$ as in the following:

$$
\overline{\boldsymbol{\alpha}}_{2}=\dot{\boldsymbol{\alpha}}_{1}-\boldsymbol{\Lambda}_{3} \mathbf{z}_{1}
$$

where $\boldsymbol{\Lambda}_{3} \in \mathbf{R}^{n \times n}$ denotes the diagonal positive definite matrix.

In addition, $\overline{\boldsymbol{\alpha}}_{2}$ is obtained using the first-order filter again as follows:

$$
\boldsymbol{\tau}_{2} \dot{\boldsymbol{\alpha}}_{2}+\boldsymbol{\alpha}_{2}=\overline{\boldsymbol{\alpha}}_{2}, \quad \boldsymbol{\alpha}_{2}(0)=\overline{\boldsymbol{\alpha}}_{2}(0),
$$

where $\boldsymbol{\tau}_{2}$ is the diagonal positive definite vector, and $\boldsymbol{\alpha}_{2}$ is the filtering control vector.

Define:

$$
\mathbf{z}_{2}=\mathbf{x}_{2}-\alpha_{2} .
$$

Substituting Eqs. (20) to (22) into Eq. (19) yields the following:

$$
\dot{\mathbf{z}}_{1}=\mathrm{z}_{2}-\tau_{2} \dot{\boldsymbol{\alpha}}_{2}-\boldsymbol{\Lambda}_{3} \mathbf{z}_{1}
$$

\section{Phase 3}

Differentiating Eq. (22) and substituting it into Eq. (8) yields:

$$
\dot{\mathbf{z}}_{2}=\dot{\mathbf{x}}_{2}-\dot{\boldsymbol{\alpha}}_{2}=\mathbf{J}^{-1} \mathbf{u}-\mathbf{J}^{-1} \mathbf{K}\left(\mathbf{x}_{1}-\mathbf{q}\right)-\dot{\boldsymbol{\alpha}}_{2} .
$$

Multiply both sides of Eq. (24) by $\mathbf{J}$,

$$
\mathbf{J} \dot{\mathbf{z}}_{2}=\mathbf{u}-\mathbf{K}\left(\mathbf{x}_{1}-\mathbf{q}\right)-\mathbf{J} \dot{\boldsymbol{\alpha}}_{2} .
$$

Thus, the actual control vector is designed as follows:

$$
\mathbf{u}=\hat{\mathbf{K}}\left(\mathbf{x}_{1}-\mathbf{q}\right)+\hat{\mathbf{J}} \dot{\boldsymbol{\alpha}}_{2}-\boldsymbol{\Lambda}_{4} \mathbf{z}_{2}
$$

where $\boldsymbol{\Lambda}_{4} \in \mathbf{R}^{n \times n}$ denotes a diagonal positive definite matrix.

By simply considering $\hat{\mathbf{K}}$ and $\hat{\mathbf{J}}$, such similar expressions as Eq. (16) are given below:

$$
\begin{aligned}
& \hat{\mathbf{K}}\left(\mathbf{x}_{1}-\mathbf{q}\right)=\operatorname{diag}\left(\mathbf{x}_{1}-\mathbf{q}\right) \hat{\boldsymbol{\theta}}_{3}, \\
& \hat{\mathbf{J}} \dot{\boldsymbol{\alpha}}_{2}=\operatorname{diag}\left(\dot{\boldsymbol{\alpha}}_{2}\right) \hat{\boldsymbol{\theta}}_{4} .
\end{aligned}
$$

From Eqs. (27) and (26), it follows that:

$$
\mathbf{u}=\operatorname{diag}\left(\mathbf{x}_{1}-\mathbf{q}\right) \hat{\boldsymbol{\theta}}_{3}+\operatorname{diag}\left(\dot{\boldsymbol{\alpha}}_{2}\right) \hat{\boldsymbol{\theta}}_{4}-\boldsymbol{\Lambda}_{4} \mathbf{z}_{2} .
$$

By substituting Eqs. (28) and (27) into Eq. (25), it follows that:

$$
\begin{aligned}
\mathbf{J} \dot{\mathbf{z}}_{2} & =(\hat{\mathbf{K}}-\mathbf{K})\left(\mathbf{x}_{1}-\mathbf{q}\right)+(\hat{\mathbf{J}}-\mathbf{J}) \dot{\boldsymbol{\alpha}}_{2}-\boldsymbol{\Lambda}_{4} \mathbf{z}_{2} \\
& =\operatorname{diag}\left(\mathbf{x}_{1}-\mathbf{q}\right) \tilde{\boldsymbol{\theta}}_{3}+\operatorname{diag}\left(\dot{\boldsymbol{\alpha}}_{2}\right) \tilde{\boldsymbol{\theta}}_{4}-\boldsymbol{\Lambda}_{4} \mathbf{z}_{2} .
\end{aligned}
$$

\subsection{Stability analysis}

Define the error-surface vectors as follows:

$$
\mathbf{s}_{i}=\boldsymbol{\alpha}_{i}-\overline{\boldsymbol{\alpha}}_{i}, \quad i=1,2 .
$$

By using Eqs. (12) and (21), Eq. (30) can be rewritten as follows:

$$
\mathbf{s}_{i}=-\boldsymbol{\tau}_{\boldsymbol{i}} \dot{\boldsymbol{\alpha}}_{\boldsymbol{i}}, \quad i=1,2 .
$$

After taking the first-order differential of Eq. (30) with respect to time and considering Eqs. (9), (20), and (31), the equations can be obtained as follows:

$$
\begin{aligned}
& \dot{\mathrm{s}}_{1}=\dot{\alpha}_{1}-\dot{\bar{\alpha}}_{1}=-\tau_{1}^{-1} \mathrm{~s}_{1}+\delta_{1}, \\
& \dot{\mathrm{s}}_{2}=\dot{\alpha}_{2}-\dot{\bar{\alpha}}_{2}=-\tau_{2}^{-1} \mathrm{~s}_{2}+\delta_{2},
\end{aligned}
$$

where $\boldsymbol{\delta}_{1}=\mathbf{d}\left(\mathbf{q}_{1}+\operatorname{diag}\left(\mathbf{u}_{r}\right) \hat{\boldsymbol{\theta}}_{2}\right) / \mathbf{d t}$ and $\boldsymbol{\delta}_{2}=\boldsymbol{\Lambda}_{3} \dot{\mathbf{z}}_{1}-\ddot{\boldsymbol{\alpha}}_{1}$ are the continuous bounded vectors.

Choose the global Lyapunov function:

$$
V=V_{1}+V_{2}+V_{3},
$$

where,

$$
\begin{aligned}
V_{1}= & \frac{1}{2} \mathbf{r}_{1}^{T} \mathbf{D}(\mathbf{q}) \mathbf{r}_{1}+\frac{1}{2} \mathbf{z}_{1}^{T} \mathbf{z}_{1}+\frac{1}{2} \mathbf{z}_{2}^{T} \mathbf{J} \mathbf{z}_{2}, \\
V_{2}= & \frac{1}{2} \mathbf{s}_{1}^{T} \mathbf{s}_{1}+\frac{1}{2} \mathbf{s}_{2}^{T} \mathbf{s}_{2}, \\
V_{3}= & \frac{1}{2} \tilde{\boldsymbol{\theta}}_{1}^{T} \boldsymbol{\Gamma}_{1}^{-1} \tilde{\boldsymbol{\theta}}_{1}+\frac{1}{2} \tilde{\boldsymbol{\theta}}_{2}^{T} \mathbf{K} \boldsymbol{\Gamma}_{2}^{-1} \tilde{\boldsymbol{\theta}}_{2} \\
& +\frac{1}{2} \tilde{\boldsymbol{\theta}}_{3}^{T} \boldsymbol{\Gamma}_{3}^{-1} \tilde{\boldsymbol{\theta}}_{3}+\frac{1}{2} \tilde{\boldsymbol{\theta}}_{4}^{T} \boldsymbol{\Gamma}_{4}^{-1} \tilde{\boldsymbol{\theta}}_{4},
\end{aligned}
$$

and $\boldsymbol{\Gamma}_{i}$ is the diagonal positive definite matrix, $i=$ $1,2,3,4$. 
Theorem. By considering the FJ robot descripted as Eqs. (1) and (2) with parametric uncertainties and the initial condition of the Lyapunov function $V(0) \leq p(p$ denotes the arbitrary positive constant), the adaptive laws can be determined using the control law (Eq. (26)) as follows:

$$
\left\{\begin{array}{l}
\dot{\hat{\boldsymbol{\theta}}}_{1}=\dot{\tilde{\boldsymbol{\theta}}}_{1}=-\boldsymbol{\Gamma}_{1} \mathbf{Y}^{T} \mathbf{r}_{1}-\eta_{1} \boldsymbol{\Gamma}_{1} \hat{\boldsymbol{\theta}}_{1} \\
\dot{\hat{\boldsymbol{\theta}}}_{2}=\dot{\tilde{\boldsymbol{\theta}}}_{2}=-\boldsymbol{\Gamma}_{2} \operatorname{diag}\left(\mathbf{u}_{\mathbf{r}}\right) \mathbf{r}_{1}-\eta_{2} \boldsymbol{\Gamma}_{2} \hat{\boldsymbol{\theta}}_{2} \\
\dot{\hat{\boldsymbol{\theta}}}_{3}=\dot{\tilde{\boldsymbol{\theta}}}_{3}=-\boldsymbol{\Gamma}_{3} \operatorname{diag}\left(\mathbf{x}_{1}-\mathbf{q}\right) \mathbf{z}_{2}-\eta_{3} \boldsymbol{\Gamma}_{3} \hat{\boldsymbol{\theta}}_{3} \\
\dot{\hat{\boldsymbol{\theta}}}_{4}=\dot{\tilde{\boldsymbol{\theta}}}_{4}=-\boldsymbol{\Gamma}_{4} \operatorname{diag}\left(\dot{\boldsymbol{\alpha}}_{2}\right) \mathbf{z}_{2}-\eta_{4} \boldsymbol{\Gamma}_{4} \hat{\boldsymbol{\theta}}_{4}
\end{array}\right.
$$

where $\eta_{i}$ represents the scalar coefficients, $\eta_{i}>0$, and $i=1,2,3,4$.

If $\boldsymbol{\Lambda}_{i}, \boldsymbol{\Gamma}_{i}, \eta_{i}(i=1,2,3,4)$ and $\tau_{i}(i=1,2)$ are selected properly, it is easy to guarantee the uniformly semi-globally boundedness of all closed-loop signals, and the tracking errors will converge to a compact set whose size can be adjusted to be arbitrarily small.

Proof. Differentiate $V_{1}$ with respect to time and simplify it based on (P3), Eqs. (17), (23), (29), and (31). Thus:

$$
\begin{aligned}
\dot{V}_{1}= & \mathbf{r}_{1}^{T} \mathbf{Y} \tilde{\boldsymbol{\theta}}_{1}+\mathbf{r}_{1}^{T} \mathbf{K} \operatorname{diag}\left(\mathbf{u}_{r}\right) \tilde{\boldsymbol{\theta}}_{2}-\mathbf{r}_{1}^{T} \boldsymbol{\Lambda}_{2} \mathbf{r}_{1} \\
& +\mathbf{r}_{1}^{T} \mathbf{K} \mathbf{z}_{1}+\mathbf{r}_{1}^{T} \mathbf{K} \mathbf{s}_{1}-\mathbf{z}_{1}^{T} \boldsymbol{\Lambda}_{3} \mathbf{z}_{1}-\mathbf{z}_{2}^{T} \boldsymbol{\Lambda}_{4} \mathbf{z}_{2} \\
& +\mathbf{z}_{1}^{T} \mathbf{z}_{2}+\mathbf{z}_{1}^{T} \mathbf{s}_{2} .
\end{aligned}
$$

Differentiate $V_{2}$ and consider Eqs. (32) and (33):

$$
\dot{V}_{2}=-\mathbf{s}_{1}^{T} \boldsymbol{\tau}_{1}^{-1} \mathbf{s}_{1}-\mathbf{s}_{2}^{T} \boldsymbol{\tau}_{2}^{-1} \mathbf{s}_{2}+\mathbf{s}_{1}^{T} \boldsymbol{\delta}_{1}+\mathbf{s}_{2}^{T} \boldsymbol{\delta}_{2} .
$$

Similar to differentiating $V_{3}$ as above:

$$
\dot{V}_{3}=\tilde{\boldsymbol{\theta}}_{1}^{T} \boldsymbol{\Gamma}_{1}^{-1} \dot{\tilde{\boldsymbol{\theta}}}_{1}+\tilde{\boldsymbol{\theta}}_{2}^{T} K \boldsymbol{\Gamma}_{2}^{-1} \dot{\tilde{\boldsymbol{\theta}}}_{2}+\tilde{\boldsymbol{\theta}}_{3}^{T} \boldsymbol{\Gamma}_{3}^{-1} \dot{\tilde{\boldsymbol{\theta}}}_{3}+\tilde{\boldsymbol{\theta}}_{4}^{T} \boldsymbol{\Gamma}_{4}^{-1} \dot{\tilde{\boldsymbol{\theta}}}_{4} .
$$

Therefore:

$$
\begin{aligned}
\dot{V}= & \dot{V}_{1}+\dot{V}_{2}+\dot{V}_{3}= \\
& -\mathbf{r}_{1}^{T} \boldsymbol{\Lambda}_{2} \mathbf{r}_{1}-\mathbf{z}_{1}^{T} \boldsymbol{\Lambda}_{3} \mathbf{z}_{1}-\mathbf{z}_{2}^{T} \boldsymbol{\Lambda}_{4} \mathbf{z}_{2}-\mathbf{s}_{1}^{T} \boldsymbol{\tau}_{1}^{-1} \mathbf{s}_{1} \\
& -\mathbf{s}_{2}^{T} \boldsymbol{\tau}_{2}^{-1} \mathbf{s}_{2}+\mathbf{r}_{1}^{T} \mathbf{K} \mathbf{z}_{1}+\mathbf{r}_{1}^{T} \mathbf{K} \mathbf{s}_{1}+\mathbf{z}_{1}^{T} \mathbf{s}_{2}+\mathbf{z}_{1}^{T} \mathbf{z}_{2} \\
& +\mathbf{s}_{1}^{T} \boldsymbol{\delta}_{1}+\mathbf{s}_{2}^{T} \boldsymbol{\delta}_{2}-\sum_{i=1, i \neq 2}^{4} \eta_{i} \tilde{\boldsymbol{\theta}}_{i}^{T} \hat{\boldsymbol{\theta}}_{i}-\eta_{2} \tilde{\boldsymbol{\theta}}_{2}^{T} \mathbf{K} \hat{\boldsymbol{\theta}}_{2} .
\end{aligned}
$$

By Young inequality, for arbitrary vector a $\in$ $\mathbf{R}^{n \times 1}, \mathbf{b} \in \mathbf{R}^{n \times 1}$ and diagonal positive definite matrix $\mathbf{H} \in \mathbf{R}^{n \times n}$, the inequality exists as follows:

$$
\mathbf{a}^{T} \mathbf{H} \mathbf{b} \leq \frac{1}{4} \mathbf{a}^{T} \mathbf{H a}+\mathbf{b}^{T} \mathbf{H} \mathbf{b} .
$$

Moreover, the set of inertia parameters $\boldsymbol{\theta}_{i}$, the estimated set $\hat{\boldsymbol{\theta}}_{i}$, and the set of estimated errors $\hat{\boldsymbol{\theta}}_{i}$ satisfy the following inequality:

$$
2 \tilde{\boldsymbol{\theta}}_{i}^{T} \hat{\boldsymbol{\theta}}_{i} \geq \tilde{\boldsymbol{\theta}}_{i}^{T} \tilde{\boldsymbol{\theta}}_{i}-\boldsymbol{\theta}_{i}^{T} \boldsymbol{\theta}_{i}
$$

By using Eqs. (42), (43) to (44), the derivative of Lyapunov function can be rewritten as follows:

$$
\begin{aligned}
\dot{V} \leq & -\mathbf{r}_{1}^{T} \boldsymbol{\Lambda}_{2} \mathbf{r}_{1}-\mathbf{z}_{1}^{T} \boldsymbol{\Lambda}_{3} \mathbf{z}_{1} \\
& -\mathbf{z}_{2}^{T} \boldsymbol{\Lambda}_{4} \mathbf{z}_{2}-\mathbf{s}_{1}^{T} \boldsymbol{\tau}_{1}^{-1} \mathbf{s}_{1}-\mathbf{s}_{2}^{T} \boldsymbol{\tau}_{2}^{-1} \mathbf{s}_{2}+\frac{1}{4} \mathbf{r}_{1}^{T} \mathbf{K} \mathbf{r}_{1} \\
& +\mathbf{z}_{1}^{T} \mathbf{K} \mathbf{z}_{1}+\frac{1}{4} \mathbf{r}_{1}^{T} \mathbf{K} \mathbf{r}_{1}+\mathbf{s}_{1}^{T} \mathbf{K} \mathbf{s}_{1}+\mathbf{z}_{1}^{T} \mathbf{z}_{1} \\
& +\frac{1}{4} \mathbf{z}_{2}^{T} \mathbf{z}_{2}+\mathbf{z}_{1}^{T} \mathbf{z}_{1}+\frac{1}{4} \mathbf{s}_{2}^{T} \mathbf{s}_{2}+\mathbf{s}_{1}^{T} \mathbf{s}_{1}+\frac{1}{4} \boldsymbol{\delta}_{1}^{T} \boldsymbol{\delta}_{1} \\
& +\mathbf{s}_{2}^{T} \mathbf{s}_{2}+\frac{1}{4} \boldsymbol{\delta}_{2}^{T} \boldsymbol{\delta}_{2}+\frac{1}{2} \sum_{i=1, i \neq 2}^{4} \eta_{i} \boldsymbol{\theta}_{i}^{T} \boldsymbol{\theta}_{i}-\frac{1}{2} \\
& \sum_{i=1, i \neq 2}^{4} \eta_{i} \tilde{\boldsymbol{\theta}}_{i}^{T} \tilde{\boldsymbol{\theta}}_{i}+\frac{1}{2} \eta_{2} \boldsymbol{\theta}_{2}^{T} \mathbf{K} \boldsymbol{\theta}_{2}-\frac{1}{2} \eta_{2} \tilde{\boldsymbol{\theta}}_{2}^{T} K \tilde{\boldsymbol{\theta}}_{2} .
\end{aligned}
$$

According to (P1) and Assumption 2, Eq. (45) is derived as follows:

$$
\begin{aligned}
\dot{V} \leq & -\mathbf{r}_{1}^{T} \frac{D(q)}{D_{M}}\left(\boldsymbol{\Lambda}_{2}-\frac{1}{2} K_{M} \mathbf{I}\right) \mathbf{r}_{1}-\mathbf{z}_{1}^{T}\left(\boldsymbol{\Lambda}_{3}-K_{M} \mathbf{I}\right. \\
& -2 \mathbf{I}) \mathbf{z}_{1}-\mathbf{z}_{2}^{T} \frac{\mathbf{J}}{J_{M}}\left(\boldsymbol{\Lambda}_{4}-\frac{1}{4} \mathbf{I}\right) \mathbf{z}_{2}-\mathbf{s}_{1}^{T}\left(\boldsymbol{\tau}_{1}^{-1}\right. \\
& \left.-K_{M} \mathbf{I}-\mathbf{I}\right) \mathbf{s}_{1}-\mathbf{s}_{2}^{T}\left(\boldsymbol{\tau}_{2}^{-1}-\frac{5}{4} \mathbf{I}\right) \mathbf{s}_{2}-\frac{1}{2} \\
& \sum_{i=1, i \neq 2}^{4} \eta_{i} \tilde{\boldsymbol{\theta}}_{i}^{T} \boldsymbol{\Gamma}_{i}^{-1} \boldsymbol{\Gamma}_{i} \tilde{\boldsymbol{\theta}}_{i}-\frac{1}{2} \eta_{2} \tilde{\boldsymbol{\theta}}_{2}^{T} K \boldsymbol{\Gamma}_{2}^{-1} \boldsymbol{\Gamma}_{2} \tilde{\boldsymbol{\theta}}_{2}+\frac{1}{2} \\
& \sum_{i=1, i \neq 2}^{4} \eta_{i} \boldsymbol{\theta}_{i}^{T} \boldsymbol{\theta}_{i}+\frac{1}{4} \boldsymbol{\delta}_{1}^{T} \boldsymbol{\delta}_{1}+\frac{1}{4} \boldsymbol{\delta}_{2}^{T} \boldsymbol{\delta}_{2}+\frac{1}{2} \eta_{2} \boldsymbol{\theta}_{2}^{T} K \boldsymbol{\theta}_{2} .
\end{aligned}
$$

Define:

$$
\begin{aligned}
& \Lambda_{2}=\frac{1}{2} K_{M} \mathbf{I}+\boldsymbol{\Lambda}_{2}^{*}, \quad \Lambda_{3}=2 \mathbf{I}+K_{M} \mathbf{I}+\Lambda_{3}^{*}, \\
& \Lambda_{4}=\frac{1}{4} I+\boldsymbol{\Lambda}_{4}^{*}, \quad \boldsymbol{\tau}_{1}^{-1}=K_{M} \mathbf{I}+\mathbf{I}+\boldsymbol{\tau}_{1}^{*}, \\
& \boldsymbol{\tau}_{2}^{-1}=\frac{5}{4} \mathbf{I}+\tau_{2}^{*},
\end{aligned}
$$

where $\boldsymbol{\Lambda}_{i}^{*}>0(i=2,3,4), \boldsymbol{\tau}_{i}^{*}>0(i=1,2)$. Therefore: 


$$
\begin{aligned}
& \dot{V} \leq-\mathbf{r}_{1}^{T} \frac{\mathbf{D}(\mathbf{q})}{D_{M}} \boldsymbol{\Lambda}_{2}^{*} \mathbf{r}_{1}-\mathbf{z}_{1}^{T} \boldsymbol{\Lambda}_{3}^{*} \mathbf{z}_{1}-\mathbf{z}_{2}^{T} \frac{\mathbf{J}}{J_{M}} \boldsymbol{\Lambda}_{4}^{*} \mathbf{z}_{2} \\
&-\mathbf{s}_{1}^{T} \boldsymbol{\tau}_{1}^{*} \mathbf{s}_{1}-\mathbf{s}_{2}^{T} \boldsymbol{\tau}_{2}^{*} \mathbf{s}_{2}-\frac{1}{2} \sum_{i=1, i \neq 2}^{4} \eta_{i} \tilde{\boldsymbol{\theta}}_{i}^{T} \\
& \boldsymbol{\Gamma}_{i}^{-1} \boldsymbol{\lambda}_{m i} \tilde{\boldsymbol{\theta}}_{i}-\frac{1}{2} \eta_{2} \tilde{\boldsymbol{\theta}}_{2}^{T} \mathbf{K} \Gamma_{2}^{-1} \boldsymbol{\lambda}_{m 2} \tilde{\boldsymbol{\theta}}_{2} \\
&+\frac{1}{2} \sum_{i=1, i \neq 2}^{4} \eta_{i} \boldsymbol{\theta}_{i}^{T} \boldsymbol{\theta}_{i}+\frac{1}{4} \boldsymbol{\delta}_{1}^{T} \boldsymbol{\delta}_{1} \\
&+\frac{1}{4} \boldsymbol{\delta}_{2}^{T} \boldsymbol{\delta}_{2}+\frac{1}{2} \eta_{2} \boldsymbol{\theta}_{2}^{T} K \boldsymbol{\theta}_{2},
\end{aligned}
$$

where $\lambda_{m i}$ is the minimum eigenvalue of $\boldsymbol{\Gamma}_{i}$.

Select the real number $\lambda$ to satisfy the inequality as follows:

$$
\begin{aligned}
0<\lambda \leq & \min \left(\frac{\left\|\boldsymbol{\Lambda}_{2}^{*}\right\|}{D_{M}},\left\|\boldsymbol{\Lambda}_{3}^{*}\right\|, \frac{\left\|\boldsymbol{\Lambda}_{4}^{*}\right\|}{J_{M}},\left\|\boldsymbol{\tau}_{1}^{*}\right\|,\left\|\boldsymbol{\tau}_{2}^{*}\right\|,\right. \\
& \left.\frac{\lambda_{m 1}}{2}, \frac{\lambda_{m 2}}{2}, \frac{\lambda_{m 3}}{2} \frac{\lambda_{m 4}}{2}\right) .
\end{aligned}
$$

Because $\delta_{1}, \delta_{2}, \theta_{1}, \theta_{2}, \theta_{3}$, and $\theta_{4}$ are bounded, the inequality can be written as follows:

$$
\frac{1}{4} \sum_{i=1}^{2} \boldsymbol{\delta}_{i}^{T} \boldsymbol{\delta}_{i}+\frac{1}{2} \sum_{i=1, i \neq 2}^{4} \eta_{i} \boldsymbol{\theta}_{i}^{T} \boldsymbol{\theta}_{i}+\frac{1}{2} \eta_{i} \boldsymbol{\theta}_{i}^{T} \mathbf{K} \boldsymbol{\theta}_{i} \leq \Phi
$$

where $\Phi$ is the constant.

Eq. (47) can be written according to Eqs. (48) and (49) as follows:

$$
\dot{V} \leq-2 \lambda V+\Phi \text {. }
$$

Consider the following compact set:

$$
\begin{aligned}
& \Omega_{1}:=\left\{\left(\mathbf{q}_{d}, \dot{\mathbf{q}}_{d}, \ddot{\mathbf{q}}_{d}\right):\left\|\mathbf{q}_{d}\right\|^{2}+\left\|\left.\dot{\mathbf{q}}_{d}\right|^{2}+\right\| \ddot{\mathbf{q}}_{d} \|^{2} \leq \chi\right\}, \\
& \Omega_{2}:=\{V \leq p\},
\end{aligned}
$$

where $p$ is an arbitrary positive constant.

Select $\lambda \geq \Phi / 2 p$. If $V=p, \dot{V} \leq 0$. Thus, $V \leq p$ presents an invariant set, i.e., if $V(0) \leq p$, then $V(t) \leq$ $p$ for all $t>0$.

Solving the Inequality Eq. (50) yields:

$$
V \leq \frac{\Phi}{2 \lambda}+\left(V(0)-\frac{\Phi}{2 \lambda}\right) e^{-2 \lambda t}
$$

Obviously, all closed-loop signals are uniformly semiglobally bounded, and:

$$
\lim _{t \rightarrow \infty} V(t) \leq \frac{\Phi}{2 \lambda} .
$$

Therefore, by adjusting parameters $\boldsymbol{\Lambda}_{i}(i=1,2,3,4)$, $\boldsymbol{\tau}_{i}(i=1,2), \boldsymbol{\Gamma}_{i}(i=1,2,3,4)$, and $\eta_{i}(i=1,2,3,4)$ to make $\lambda$ bigger, the tracking error will be smaller.

\section{Simulation results and analysis}

The two-link FJ robot shown in Figure 1 with parametric uncertainties is used to test the feasibility of the proposed control method. The dynamic equations are derived based on Kane method, and numerical calculation is finished in Simulink of MATLAB.

The robot dynamic parameters are given as follows:

$$
\begin{aligned}
& m_{1}=6.07, \quad m_{2}=5.76, \\
& \mathbf{p}_{c 1}=(0.169,0,0.0026)^{T}, \quad \mathbf{p}_{c 2}=(0.162,0,0)^{T}, \\
& \mathbf{p}_{01}=(0,0,0.055)^{T}, \quad \mathbf{p}_{12}=(0.3,0,0.06)^{T} .
\end{aligned}
$$

Here, $m_{i}(i=1,2)$ is the $i$-th link mass, and $\mathbf{p}_{c i}(i=$ $1,2)$ is the mass center of the $i$-th link relative to the $i$-th link coordinate. $\mathbf{p}_{i-1, i}(i=1,2)$ is the relative position between the $i$-th joint and $i-1$-th joint. In addition,

$$
\begin{aligned}
& \mathbf{I}_{c 1}=\left[\begin{array}{ccc}
0.0074 & 0 & -0.002 \\
0 & 0.0718 & 0 \\
-0.002 & 0 & 0.0742
\end{array}\right], \\
& \mathbf{I}_{c 2}=\left[\begin{array}{ccc}
0.0065 & 0 & 0 \\
0 & 0.0654 & 0 \\
0 & 0 & 0.0685
\end{array}\right], \\
& \mathbf{J}=\operatorname{diag}(0.0197,0.0197), \\
& \mathbf{g}=(0,-9.8,0)^{T}, \\
& K_{1}=K_{2}=100 .
\end{aligned}
$$

Now, the minimum inertial parameters can be solved as follows:

$$
\begin{aligned}
& \boldsymbol{\theta}_{1}=(2.84,0,0.766,0.9331,0,0.2197)^{T}, \\
& \boldsymbol{\theta}_{2}=(0.01,0.01)^{T}, \\
& \boldsymbol{\theta}_{3}=(100,100)^{T} \\
& \boldsymbol{\theta}_{4}=(0.0197,0.0197)^{T} .
\end{aligned}
$$

The ideal trajectories are given as $q_{1 d}=\sin (2 \pi t)$ and

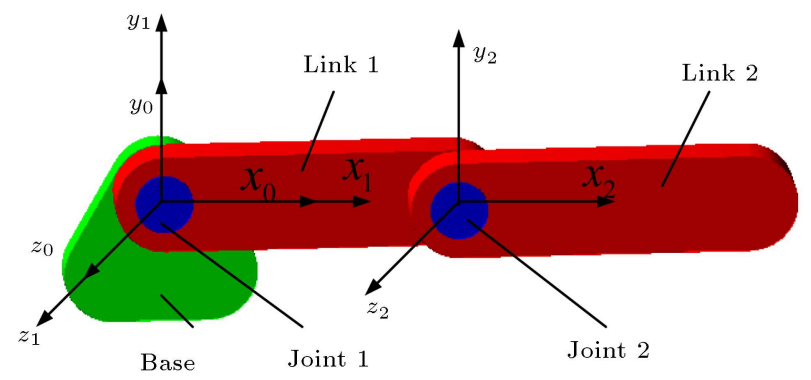

Figure 1. 3D model of two-link FJ robot. 
$q_{2 d}=\sin (2 \pi t)$; the initial positions are specified as $q_{1}(0)=q_{2}(0)=0, q_{m 1}(0)=q_{m 2}(0)=0$, and the controller parameters are chosen by Eq. (46) as follows:

$$
\begin{aligned}
& \eta_{1}=\eta_{2}=\eta_{3}=\eta_{4}=0.0005, \\
& \tau_{1}=\boldsymbol{\tau}_{2}=\operatorname{diag}(0.001,0.001) \\
& \boldsymbol{\Gamma}_{1}=\boldsymbol{\Gamma}_{2}=\boldsymbol{\Gamma}_{3}=\Gamma_{4}=\operatorname{diag}(0.00005,0.00005), \\
& \Lambda_{1}=\operatorname{diag}(60,60), \quad \Lambda_{2}=\operatorname{diag}(60,60) \\
& \Lambda_{3}=\operatorname{diag}(150,150), \quad \Lambda_{4}=\operatorname{diag}(5,5) .
\end{aligned}
$$

The initial estimated conditions of the inertia parameters are set as: $\hat{\boldsymbol{\theta}}_{1}(0)=(0,0,0,0,0,0)^{T}, \hat{\boldsymbol{\theta}}_{2}(0)=$ $(0,0)^{T}, \hat{\boldsymbol{\theta}}_{3}(0)=(0,0)^{T}$, and $\hat{\boldsymbol{\theta}}_{4}(0)=(0,0)^{T}$.

The tracking trajectories of Links 1 and 2 are placed in Figure 2(a) and (b) together with their ideal trajectories. The simulation results are shown in Figure 2(c) and (d). These figures show that the proposed control method results in the very small link tracking errors $\left(e_{1}\right.$ and $e_{2}$ are both about $5 \%$ of the ideal trajectories) and ensures the global boundedness of all closed-loop signals with uncertainties.

1. Uncertainties of the initial values: The initial values of $\hat{\boldsymbol{\theta}}_{i}$ are not restrained theoretically, that is, they can be selected arbitrarily. For verifying their effect, the other parameters should be kept unchanged and the initial values of $\hat{\boldsymbol{\theta}}_{i}$ are set to $0 \%$, $50 \%$, and $80 \%$, respectively, of the nominal value $\boldsymbol{\theta}_{i}$ as follows:

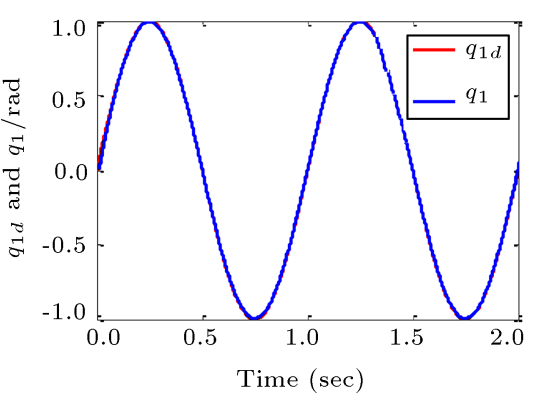

(a)

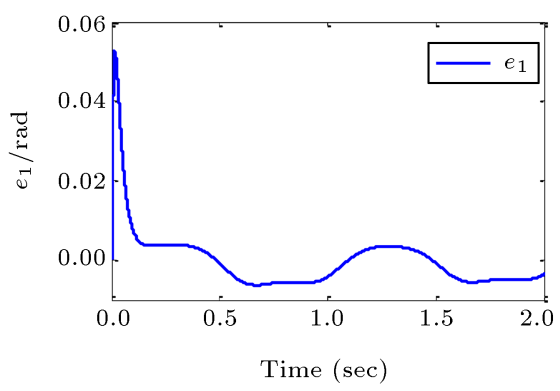

(c)
(1) $\hat{\boldsymbol{\theta}}_{1}=(0,0,0,0,0,0)^{T}, \quad \hat{\boldsymbol{\theta}}_{2}=(0,0)^{T}$, $\hat{\boldsymbol{\theta}}_{3}=(0,0)^{T}, \quad \hat{\boldsymbol{\theta}}_{4}=(0,0)^{T}$,

(2) $\hat{\boldsymbol{\theta}}_{1}=(1.42,0,0.38,0.47,0,0.11)^{T}$, $\hat{\boldsymbol{\theta}}_{2}=(0.005,0.005)^{T}, \quad \hat{\boldsymbol{\theta}}_{3}=(50,50)^{T}$, $\hat{\boldsymbol{\theta}}_{4}=(0.01,0.01)^{T}$,

(3) $\hat{\boldsymbol{\theta}}_{1}=(2.27,0,0.61,0.75,0,0.18)^{T}$, $\hat{\boldsymbol{\theta}}_{2}=(0.008,0.008)^{T}, \quad \hat{\boldsymbol{\theta}}_{3}=(80,80)^{T}$, $\hat{\boldsymbol{\theta}}_{4}=(0.016,0.016)^{T}$.

The tracking errors in the simulation are shown in Figure 3(a) and (b). They reveal that the tracking errors of Links 1 and 2 respectively decrease as the estimated values are closer to the nominal value of $\boldsymbol{\theta}_{i}$, and the their simulation computing times are equal almost. Further, the tracking errors can be lower if the estimated values of inertia parameters are closer to the nominal values.

2. Uncertainties of parameters of the control laws: For verifying the effectiveness of the proposed adaptive laws, we change these parameters continuously as:

$\boldsymbol{\Gamma}_{1}=\boldsymbol{\Gamma}_{2}=\boldsymbol{\Gamma}_{3}=\boldsymbol{\Gamma}_{4}=\operatorname{diag}(0.00005,0.00005) * \sin (\mathbf{t})$

$\eta_{1}=\eta_{2}=\eta_{3}=\eta_{4}=0.0005 * \sin (\mathbf{t})$.

The tracking errors are shown in Figure 4(a) and (b), in which $e_{1}$ and $e_{2}$ represent the tracking errors of Links 1 and 2, respectively, with the original adaptive laws parameters; $e_{11}$ and $e_{22}$ represent the errors with the changed parameters.

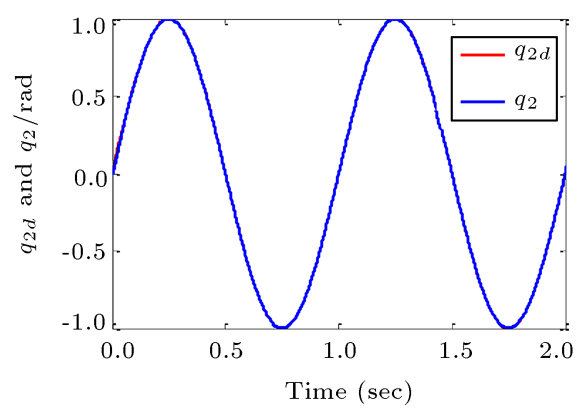

(b)

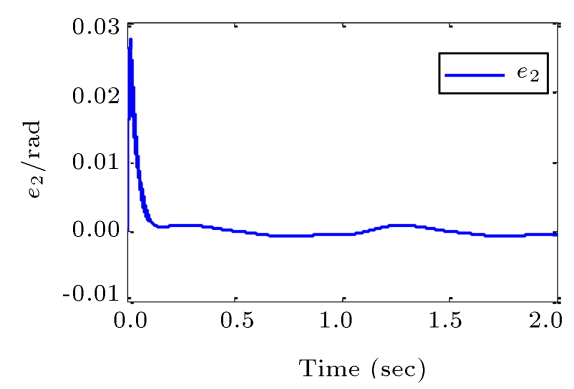

(d)

Figure 2. The trajectories and tracking errors of Links 1 and 2 with the designed parameters: (a) Ideal and tracking trajectory of Link 1, (b) ideal and tracking trajectory of Link 1, (c) errors of Link 1, and (d) errors of Link 2. 


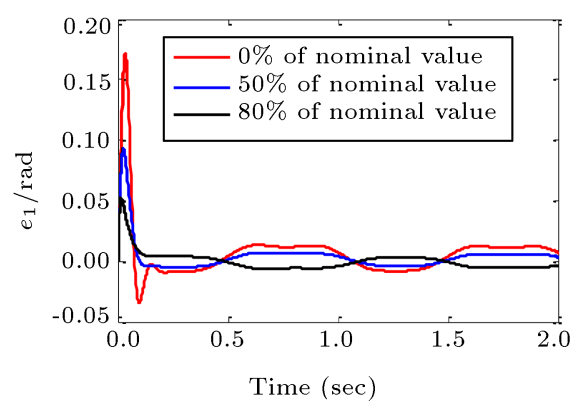

(a)

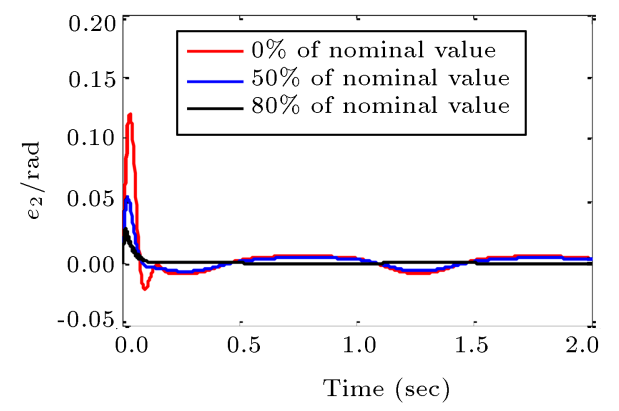

(b)

Figure 3. The tracking errors of Links 1 and 2 with the different initial values of $\hat{\boldsymbol{\theta}}_{i}(i=1,4)$ : (a) Errors of Link 1 and (b) errors of Link 2.

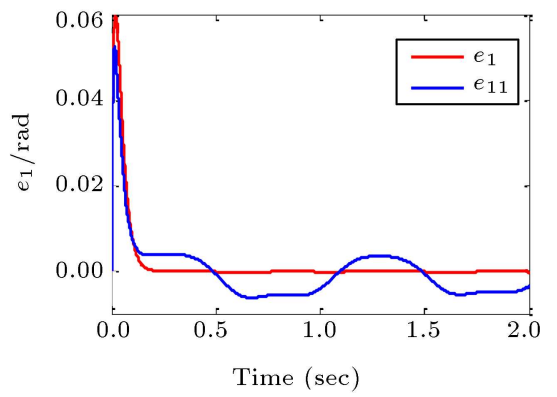

(a)

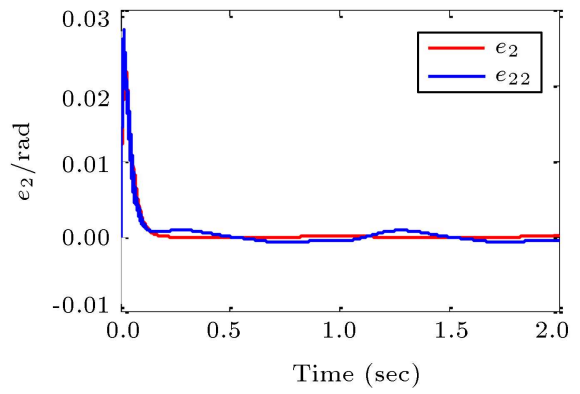

(b)

Figure 4. The tracking errors of Link 1 with different parameters of the control laws: (a) Errors of Link 1 with the original and changed parameters and (b) errors of Link 2 with the original and changed parameters.

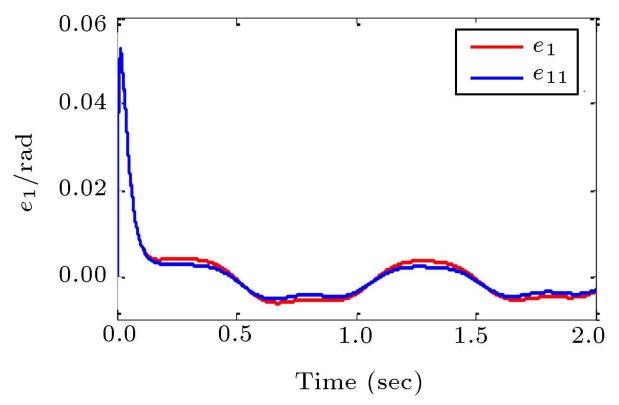

(a)

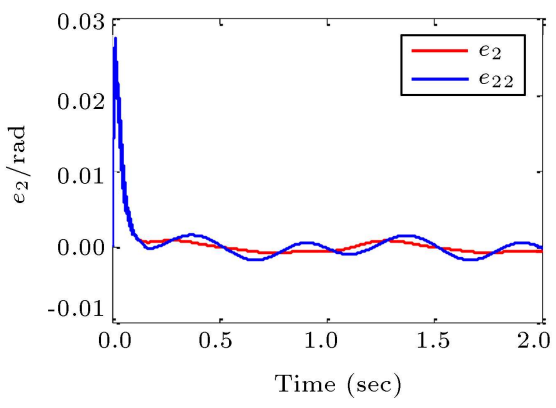

(b)

Figure 5. The tracking errors of Link 1 with different external disturbances: (a) Errors of Link 1 without and with external disturbance and (b) errors of Link 2 without and with external disturbance.

It is evident that the tracking errors of Links 1 and 2 are kept small, even though the parameters of adaptive laws are changed.

3. Uncertainties of external disturbances: Add the external disturbances on the joints of Links 1 and 2 with functions $50 \sin (2 \pi \mathbf{t})$ and $35 \sin (4 \pi \mathbf{t})$, respectively.

The tracking errors are shown in Figure 5(a) and (b), in which $e_{1}$ and $e_{2}$ represent the tracking errors of Links 1 and 2, respectively, without the external disturbances; $e_{11}$ and $e_{22}$ represent the errors with the external disturbances.

It is clear that the external disturbances cause the tracking errors of the links slightly, and tracking errors become weak quickly in $0.1 \mathrm{sec}$.

In addition, the errors of links are less than $15 \%$ of desired amplitude in the beginning phase whether the inertia parameters, control laws parameters or external disturbance are changed or not. In literature [14], we investigated an adaptive backstepping control method based on NN; the maximum tracking errors of links are more than $20 \%$ of input amplitude, and timeconsumption is at least twice what this paper suggests.

\section{Conclusions}

In this paper, the uncertainties associated with the 
robot model were estimated by using the inertia parameters of FJ robots. The adaptive DSC for FJ robot was proposed based on backstepping control method, in which the virtual control vectors were derived by using the first-order filter in order to avoid the "explosion of derivative". The control vectors and adaptive laws in the DSC were expressed as the functions of inertia parameters in FJ robots. In addition, the joint tracking errors could be very insignificant, even though the initial estimated conditions of the inertia parameters were set to be far away from the real ones, the parameters of the control laws were changed, or the external disturbances were added in the system.

The simulations of the two-link FJ robot showed that the proposed control system achieved:

1. Less tracking error, better tracking performance and robustness against model uncertainties;

2. Simpler calculation to avoid high-order derivative and iterative regression matrix;

3. Shorter computation time to respond;

4. Easier selection of control parameters.

Moreover, the tracking performance in a short period of time can be better if the inertia parameters are selected precisely.

\section{Contributors}

Chenggang Li proposed the adaptive dynamic surface control applied to the flexible-joint robot and derived the adaptation laws. Wen Cui carried out the simulation and calculation. Dengdeng Yan studied the control method based on Neural network and discussed the uncertainties of FJ robots. Yan Wang built the dynamics of robot. Chunming Wang verified the dynamics and simulation in the paper about the adaptive control.

\section{Acknowledgement}

This work is supported by the Natural Science Foundation of Jiangsu Province under Grant No. BK20141414.

\section{Nomenclature}

$\mathbf{q}, \dot{\mathbf{q}}, \ddot{\mathbf{q}} \quad$ Position, velocity, and acceleration vectors of the links

$\mathbf{D}(\mathbf{q}) \quad$ Inertia matrix

$\mathbf{C}(\mathbf{q}, \dot{\mathbf{q}}) \quad$ Coriolis and centripetal matrix;

$\mathbf{G}(\mathbf{q}) \quad$ Gravity matrix

K Positive definite diagonal constant flexibility matrix

$\mathbf{q}_{\mathbf{m}}, \ddot{\mathbf{q}}_{\mathbf{m}} \quad$ Position and acceleration vectors for the actuator
$\mathbf{J}$

$\mathbf{u}$

$D_{m}, D_{M}$

$C_{M}, G_{M}$

$\mathbf{Y}(\mathbf{q}, \dot{\mathbf{q}}, \ddot{\mathbf{q}})$

$\boldsymbol{\theta}_{1}$

$r$

$\mathbf{a}_{1}, \boldsymbol{v}_{1}$

$\mathbf{x}_{1}, \mathbf{x}_{2}$

$K_{m}, K_{M}$

$J_{m}, J_{M}$

$\mathbf{q}_{d}$

$Q$

$\overline{\boldsymbol{\alpha}}_{1}, \overline{\boldsymbol{\alpha}}_{2}$

$\alpha_{1}, \alpha_{2}$

$\mathbf{u}_{r}$

$\boldsymbol{\Lambda}_{1}, \boldsymbol{\Lambda}_{2}, \boldsymbol{\tau}_{1}$

$\boldsymbol{\Lambda}_{3}, \boldsymbol{\Lambda}_{4}, \boldsymbol{\tau}_{2}, \mathbf{H}$

$\delta_{1}, \delta_{2}$

$\Gamma_{i}$

$\eta_{i}$

$\mathbf{a}, \mathbf{b}$

$\boldsymbol{\theta}_{i}, \hat{\boldsymbol{\theta}}_{i}, \tilde{\boldsymbol{\theta}}_{i}$

$\lambda_{m i}$

$\lambda$

$\Phi$

$p$

$m_{i}(i=1,2) \quad i$-th link mass

$\mathbf{p}_{c i}(i=1,2) \quad$ Mass centers of the $i$-th link relative to the $i$-th link coordinate

$\mathbf{p}_{i-1, i}(i=1,2)$ Relative position between the $i$-th joint and the $i$-1-th joint

$q_{1 d}, q_{2 d} \quad$ Ideal trajectories

$e_{1}, e_{2} \quad$ Link tracking errors

\section{References}

1. Chen, Z.Y. and Chen, L. "Adaptive backstepping control of flexible-joint space robot based on neural network", Engineering Mechanics, 30(4), pp. 397-401 (2013).

2. Macnab, C.J.B. and D'Eleuterio, G.M.T. "Neuroadaptive control of elastic-joint robots using robust performance enhancement", Robotica, 19(6), pp. 619-629 (2001).

3. Nanos, K. and Papadopoulos, E. "On the use of free-floating space robots in the presence of angular momentum", Intelligent Service Robotics, 4(1), pp. 315 (2011). 
4. Karabacak, M. and Eskikurt, H.I. "Design, modelling and simulation of a new nonlinear and full adaptive backstepping speed tracking controller for uncertain PMSM", Applied Mathematical Modelling, 36(11), pp. 5199-5213 (2012).

5. Karabacak, M. and Eskikurt, H.I. "Speed and current regulation of a permanent magnet synchronous motor via nonlinear and adaptive backstepping control", Mathematical \& Computer Modelling, 53(9-10), pp. 2015-2030 (2011).

6. Swaroop, D., Hedrick, J.K., Yip, P.P., and Gerdes, J.C. "Dynamic surface control for a class of nonlinear systems", IEEE Transactions on Automatic Control, 45(10), pp. 1893-1899 (2002).

7. Wu, Z.H., Lu, J.C., and Shi, J.P. "Adaptive neural dynamic surface control of morphing aircraft with input constraints", 29th Chinese Control and Decision Conference, Chongqing, China, pp. 6-12 (2017).

8. Zhang, T., Xia, M., Yi, Y., and Shen, Q. "Adaptive neural dynamic surface control of pure-feedback nonlinear systems with full state constraints and dynamic uncertainties", IEEE Transactions on Systems Man Cybernetics-Systems, 47(8), pp. 2378-2387 (2017).

9. Wang, C., Wu, Y., and Yu, J. "Barrier Lyapunov functions-based dynamic surface control for purefeedback systems with full state constraints", IET Control Theory and Applications, 11(4), pp. 524-530 (2017).

10. Shin, J. "Adaptive dynamic surface control for a hypersonic aircraft using neural networks", IEEE Transactions on Aerospace and Electronic Systems, 53(5), pp. 2277-2289 (2017).

11. Gao, S.G., Dong, H.R., Ning, B., Tang, T., Li, Y.D., and Valavanis, K.P. "Neural adaptive dynamic surface control for mismatched uncertain nonlinear systems with nonlinear feedback errors", 36th Chinese Control Conference (CCC). Dalian, China, pp. 828-833 (2017).

12. Min, W. and Huiping, Y. "Adaptive neural dynamic surface control for flexible joint manipulator with prescribed performance", 29th Chinese Control and Decision Conference, Chongqing, China, pp. 53115316 (2017).

13. Uyen, H.T.T., Tuan, P.D., Tu, V.V., Quang, L., and Minh, P.X. "Adaptive neural networks dynamic surface control algorithm for 3 DOF surface ship", International Conference on System Science and Engineering, Ho Chi Minh City, Vietnam, pp. 71-76 (2017).

14. Li, C., Cui, W., You, J., Lin, J., and Xie, Z. "Neural network adaptive backstepping control of multi-link flexible-joint robots", Journal of Shanghai Jiaotong University, 50(7), pp. 1095-1101 (2016) (In Chinese).

15. Zhai, D., Xi, C., An, L., Dong, J., and Zhang, Q. "Prescribed performance switched adaptive dynamic surface control of switched nonlinear systems with average dwell time", IEEE Transactions on Systems Man Cybernetics-Systems, 47(7), pp. 1257-1269 (2017).
16. Su, H. and Zhang, W. "A combined backstepping and dynamic surface control to adaptive fuzzy statefeedback control", International Journal of Adaptive Control and Signal Processing, 31(11), pp. 1666-1685 (2017).

17. Gao, S., Dong, H., Ning, B., and Yao, X. "Singleparameter-learning-based fuzzy fault-tolerant output feedback dynamic surface control of constrained-input nonlinear systems", Information Sciences, 385, pp. 378-394 (2017).

18. Edalati, L., Edalati, L., Sedigh, A.K., Shooredeli, M.A., and Moarefianpour, A. "Adaptive fuzzy dynamic surface control of nonlinear systems with input saturation and time-varying output constraints", $M e$ chanical Systems and Signal Processing, 100, pp. 311329 (2018).

19. Rahmani, S. and Shahriari-kahkeshi, M. "Adaptive dynamic surface control design for a class of uncertain nonlinear systems using interval type-2 fuzzy systems", Iranian Conference on Electrical Engineering, Tehran, Iran, pp. 817-822 (2017).

20. Liu, X., Su, C.Y., and Yang, F. "FNN approximationbased active dynamic surface control for suppressing chatter in micro-milling with piezo-actuators", IEEE Transactions on Systems Man Cybernetics-Systems, 47(8), pp. 2100-2113 (2017).

21. Zhu, Q., Ma, J., Liu, Z., and Liu, K. "Containment control of autonomous surface vehicles: A nonlinear disturbance observer-based dynamic surface control design", Advances in Mechanical Engineering, 9(10), pp. 1-13 (2017).

22. Zhou, C., Zhu, J., Lei, H., and Yuan, X. "Observerbased dynamic surface control for high-performance aircraft subjected to unsteady aerodynamics and actuator saturation", Proceedings of the Institution of Mechanical Engineers Part I-Journal of Systems and Control Engineering, 231(6), pp. 481-494 (2017).

23. Dian, S., Chen, L., Son, H., Zhao, T., and Tan, J. "Gain scheduled dynamic surface control for a class of underactuated mechanical systems using neural network disturbance observer", Neurocomputing, 275, pp. 1998-2008 (2018).

24. Yu, Z., Qu, Y., and Zhang, Y. "Robust adaptive dynamic surface control for receiver UAV during boom refueling in the presence of vortex", 29th Chinese Control and Decision Conference, Chongqing, China, pp. 1798-1803 (2017).

25. Li, X., Wang L., and Sun, Y. "Dynamic surface backstepping sliding mode position control of permanent magnet linear synchronous motor", IEEE International Electric Machines and Drives Conference, Miami, FL, USA, pp. 1-7 (2017).

26. Chen, Z., Lin, Z., Huang, G., Jia, H., and Yue, C. "Particle swarm optimized adaptive dynamic surface control for PMSM servo system", 36th Chinese Control Conference (CCC), Dalian, China, pp. 4751-4756 (2017). 
27. Liang, T., Wang, W., Wu, S., and Lu, K. "Nonlinear attitude control of tiltrotor aircraft based on dynamic surface adaptive backstepping", 29th Chinese Control and Decision Conference, Chongqing, China, pp. 603608 (2017).

28. Yoo, S.J., Jin, B.P., and Choi, Y.H. "Adaptive output feedback control of flexible-joint robots using neural networks: dynamic surface design approach", IEEE Trans Neural Networks, 19(10), pp. 1712-1726 (2008).

29. Ortega, R. and Spong, M.W. "Adaptive motion control of rigid robots: a tutorial", Automatica, 25(6), pp. 877-888 (1989).

\section{Biographies}

Chenggang Li received the BSc degree in Hydraulic Transmission and Control from Jilin University of Technology, Changchun in 1998 and obtained the MSc and $\mathrm{PhD}$ degrees in Mechanical and Electrical Engineering from Jilin University, Changchun, 2001 and Beijing Institute of Technology, 2004, respectively. $\mathrm{He}$ is currently an Associate Professor of Mechanical and Electrical Engineering with the Nanjing University of Aeronautics and Astronautics. His major research activities are system design and industrial robot, tissue modeling and contact analysis, multidimensional sensor design, and parallel mechanism.

Wen Cui received the BSc degree in Mechanical and Electrical Engineering from Nanjing Forestry Univer- sity in 2013; then, he obtained the MSc degree in Mechanical and Electrical Engineering from Nanjing University of Aeronautics and Astronautics, Nanjing in 2016. His research interests include nonlinear control and industrial robot design.

Dengdeng Yan received the BSc degree in Mechanical Engineering from Zhejiang University of Technology in 2015. He is currently pursuing the MSc degree in Mechanical and Electrical Engineering with Nanjing University of Aeronautics and Astronautics, Nanjing. His current research interests include error analysis and compensation of manipulator.

Yan Wang received the BSc degree in Mechanical Engineering from China Three Gorges University in 2015. She is currently pursuing the MSc degree in Mechanical and Electrical Engineering with Nanjing University of Aeronautics and Astronautics, Nanjing. Her current research interests include structure design and dynamics of manipulator.

Chunming Wang received the BSc degree in Mechanical Engineering from Nanjing University of Aeronautics and Astronautics in 2016. He is currently pursuing the MSc degree in Mechanical and Electrical Engineering with Nanjing University of Aeronautics and Astronautics, Nanjing. His current research interests include active compliance control and nonlinear control of robot. 